\title{
Trimodality therapy in stage III non-small cell lung cancer: prediction of recurrence by assessment of $185^{\text {neu }}$
}

\author{
M. Thomas*, C. Rübe**, M. Semik', M. von Eiff*, F. Klinke ${ }^{++}$, H.N. Macha ${ }^{\S}$, L. Freitag ${ }^{\S}$, \\ H.H. Scheld ${ }^{+}$, N. Willich**, W.E. Berdel*, K. Junker ${ }^{\S \S}$
}

Trimodality therapy in stage III non-small cell lung cancer: prediction of recurrence by assessment of $185^{\text {neu }}$. M. Thomas, C. Rübe, M. Semik, M. von Eiff, F. Klinke, H.N. Macha, L. Freitag, H.H. Scheld, N. Willich, W.E. Berdel, K. Junker. (C)ERS Journals Ltd 1999. ABSTRACT: In a trimodality treatment approach for stage III non-small cell lung cancer the prognostic impact of pretherapeutic $\mathrm{p} 185^{\text {neu }}$ assessment was evaluated.

Fifty-four patients were admitted to chemotherapy followed by twice-daily radiation with concomittant low-dose chemotherapy and subsequent surgery. Immunohistochemical assessment of $\mathbf{p 1 8 5}^{\mathrm{neu}}$ expression was performed in paraffin-embedded mediastinal lymph node metastases, by mediastinoscopy biopsy prior to therapy.

Paraffin-embedded biopsies of mediastinal lymph node metastases were available in 33 cases. Seven out of eight patients with positive $\mathbf{p 1 8 5}^{\text {neu }}$ staining developed distant metastases, in contrast to seven out of 25 negative cases. Expression of p185 $^{\text {neu }}$ in mediastinal lymph node metastases was a significant predictor for progression-free survival $(p=0.047)$ and resulted mainly from significant differences in metastases-free survival (p185 ${ }^{\text {neu}}-$ positive versus p185 $^{\text {neu }}$-negative: median, 11 versus 19 months; 2and $3-y r$ rates, $13 \%$ and $0 \%$ versus $40 \%$ and $32 \% ; p=0.04$ ).

On the basis of these preliminary results it was concluded that further evaluation of $\mathbf{p 1 8 5}^{\text {neu }}$ expression in trials on neoadjuvant and adjuvant therapy is warranted. When the prognostic impact of $\mathbf{p 1 8 5}^{\text {neu }}$ in such trials with larger patient numbers is confirmed, this may contribute to the identification of stratification variables for future treatment approaches of non-small cell lung cancer.

Eur Respir J 1999; 13: 424-429.
Depts of *Hematology/Oncology and Respiratory Medicine, **Radiation Oncology, ThoracicandCardiovascularSurgery,Westfälische-Wilhelms Universität, Münster, Germany. ${ }^{++}$Dept of Thoracic Surgery, St. Elisabeth Hospital, Ibbenbüren, Germany. ${ }^{5}$ Lungenklinik Hemer, Hemer, Germany. ${ }^{\S \S}$ Institute of Pathology, University Hospital Bergmannsheil, Bochum, Germany.

Correspondence: M. Thomas

Medizinische Klinik A

University of Münster

Albert-Schweitzer Str. 33

D-48129 Münster

Germany

Fax: 492518347680

Keywords: Non-small cell lung cancer $\mathrm{p} 185^{\text {neu }}$

prognostic factor

trimodality therapy

Received: April 231998

Accepted after revision September 241998
Conventional therapy of stage III non-small cell lung cancer (NSCLC) by surgical resection or radiation alone results in survival rates of 5-13\% [1]. These poor survival rates can be improved significantly by multimodality treatment using neoadjuvant chemotherapy or chemoradiotherapy followed by surgery or chemotherapy with simultaneous or sequential radiotherapy [2]. Previous studies on neoadjuvant therapy have demonstrated that the degree of tumour regression in resection specimens and lymph nodes is suggestive for long-term survival in these patients [2-4]. However, as the treatment-related mortality rates ranged $5-15 \%$ [2-4], it would be important to identify patients prior to treatment who are most likely to benefit from this therapy.

In the last few years, several molecular genetic features have been considered as powerful determinants of prognosis in NSCLC, especially in resectable stages of disease [5]. Recently, in patients who underwent neoadjuvant chemotherapy followed by surgery, a retrospective analysis assessed aberrant p53 expression in mediastinal lymph node metastases from biopsies taken prior to therapy. Results indicated a significant association between aberrant p53 expression and a lower degree of tumour regression in resection specimens. No correlations with survival or progression-free survival were reported [6]. The present study investigated $\mathrm{p} 185^{\text {neu }}$ overexpression in mediastinal lymph node metastases prior to multimodality treatment in stage III NSCLC patients.

The c-erbB-2-proto-oncogene encoded $\mathrm{p} 185^{\text {neu }}$ is a transmembrane glycoprotein with intrinsic tyrosine kinase activity and extensive sequence homology to the epidermal growth factor receptor. It plays an important role in regulating epithelial cell growth. Overexpression of $\mathrm{p} 185^{\text {neu }}$ has been reported in up to $30 \%$ of NSCLCs [7-10]. In patients with resectable disease, several reports point to overexpression of $\mathrm{p} 185^{\text {neu }}$ as being associated with a significantly shorter survival $[8,9,11,12]$ or disease-free survival $[12,13]$ and to be an independent prognostic factor for unfavourable postoperative outcome [8, 11-13]. This study evaluated the prognostic impact of $\mathrm{p} 185^{\text {neu }}$ overexpression in mediastinal lymph node metastases for the first time in stage III NSCLC patients prior to neoadjuvant multimodality treatment.

\section{Patients and methods}

\section{Study subjects}

The study was approved by the Ethics Committee of the University of Münster. Patients with stage IIIA [T1-3 N2 
M0] NSCLC with histologically confirmed N2 status or stage IIIB [T4 N1-3 M0/T1-4 N3 M0] NSCLC were admitted after written informed consent. Patients with involvement of supraclavicular lymph nodes or positive pleural effusion were not eligible. Further requirements were a favourable medical condition (Eastern Cooperative Oncology Group (ECOG) performance score 0,1), age of 18$69 \mathrm{yrs}$, sufficient bone marrow reserve (leukocytes $>4,000$ cells $\mu \mathrm{L}^{-1}$; thrombocytes $>100,000$ cells $\left.\mu \mathrm{L}^{-1}\right)$, adequate liver and kidney function (bilirubin $<1.5 \mathrm{mg} \cdot \mathrm{dL}^{-1} /$ creatinine $<1.5 \mathrm{mg} \cdot \mathrm{dL}^{-1}$; creatinine clearance $>30 \mathrm{~mL} \cdot \mathrm{min}^{-1}$ ) and a predicted postoperative forced expiratory volume in one second (FEV1) of $>1.0$ L. Pretreatment evaluation included laboratory profile, bronchoscopy and image diagnosis, with chest radiograph, computed tomography (CT) scans of thorax, abdomen, and head, and a bone scan. Mediastinoscopy was performed to assess the mediastinal lymph node status. Patients were also enrolled after assessment of mediastinal lymph nodes by an exploratory thoracotomy. Between April 1992 and September 1995, a total of 54 patients were enrolled from four participating institutions. As paraffin-embedded biopsies of the primary tumour were mostly used up by the primary pathologist and the study's reference pathology centre for histological confirmation of diagnosis, paraffin-embedded mediastinal lymph node metastases were procured for pretherapeutic p185 $5^{\text {neu }}$ assessment. Forty-six patients underwent surgical staging of mediastinal lymph nodes. Paraffin-embedded lymph node biopsies from 40 patients were available for histomorphological assessment. In seven out of 40 specimens, either the paraffin blocks available for examination revealed lymphatic tissue without a tumour, or the embedded tissue had been used up completely by the primary pathologist. From 33 patients, paraffin-embedded lymph node biopsies contained tumour tissue and were analysed for $\mathrm{p} 185^{\mathrm{neu}}$ expression. This patient cohort was the subject of further analysis (table 1).

\section{Treatment}

Treatment consisted of two initial cycles of chemotherapy (second cycle started on day 22) with carboplatin 300 $\mathrm{mg} \cdot \mathrm{m}^{-2}$ (day 1); ifosfamide $1,500 \mathrm{mg} \cdot \mathrm{m}^{-2}$ (days $1,3,5$ ); and etoposide $100 \mathrm{mg} \cdot \mathrm{m}^{-2}$ (days $\left.1,3,5\right)$. Granulocyte colony stimulating factor $(300 \mu \mathrm{g})$ was given daily subcutaneously starting on day 6 until the leukocyte count exceeded 4,000 cells $\mu \mathrm{L}^{-1}$. Three weeks after the start of the second cycle of chemotherapy, concurrent chemo- and radiotherapy were commenced. Two 1.5 -Gy fractions per day, with an intertreatment interval of at least $6 \mathrm{~h}$, were administered 5 days. week $^{-1}$ to a total dose of 45 Gy (days 43-61). The target volume included the primary lesion with margins of $1.5 \mathrm{~cm}$, and the ipsilateral hilum and ipsilateral mediastinum extending inferiorly $5 \mathrm{~cm}$ below the tracheal bifurcation with a margin of $0.5-1 \mathrm{~cm}$. For N3 disease, the contralateral mediastinal lymph nodes, but not the contralateral hilum, were included with margins of 0.5 $\mathrm{cm}$. Carboplatin $\left(100 \mathrm{mg} \cdot \mathrm{m}^{-2}\right)$ and vindesine $(3 \mathrm{mg}$ absolute) were administered on days 43,50 , and 57 . Five to 8 weeks after completion of combined chemoradiotherapy, patients who remained free of distant metastases were eligible for thoracotomy, with the aim of achieving complete resection of the tumour (resection margin microscopically
Table 1. - Pretreatment characteristics of patients with appropriate biopsies of mediastinal lymph node metastases for immunohistochemistry $(n=33)$

\begin{tabular}{lcc}
\hline Characteristic & $\begin{array}{c}\text { Number of } \\
\text { patients }\end{array}$ & Percentage \\
\hline Age yrs & \multicolumn{2}{c}{57} \\
Median & \multicolumn{2}{c}{$37-67$} \\
Range & \multicolumn{2}{c}{$30: 3$} \\
Male:female & \multicolumn{2}{c}{} \\
Performance status (ECOG) & 21 & 64 \\
0 & 12 & 36 \\
1 & 24 & 73 \\
Histologic type & 9 & 27 \\
Squamous type & & \\
Adenocarcinoma & 20 & 61 \\
TNM stage & 13 & \\
Total stage IIIA & 7 & 39 \\
T2N2M0 & 13 & \\
T3N2M0 & 4 & \\
Total stage IIIB & 4 & \\
T4N2M0 & 5 & \\
T2N3M0 & & \\
T3N3M0 & & \\
\hline
\end{tabular}

ECOG: Eastern Cooperative Oncology Group; TNM: tumour node metastasis.

free of tumour cells) and extensive mediastinal lymph node sampling. Patients who had an incomplete tumour resection or who were inoperable received further radiotherapy with conventional fractionation $(16 \mathrm{~Gy} ; 5 \times 2$ $\mathrm{Gy} \cdot \mathrm{week}^{-1}$ ). The target volume included the primary lesion or the bronchial stump, the ipsilateral hilum and the mediastinum.

\section{Response assessment and follow-up}

Prior to chemoradiotherapy the response to the initial chemotherapy was assessed by a thoracal CT scan and abdominal sonography. Preoperative assessment comprised laboratory profile, bronchoscopy, chest radiograph, bone scan, and CT scans of thorax, abdomen and head. The clinical response was assessed according to the guidelines of the South-West Oncology Group (SWOG) [14]. Followup examinations (laboratory profile, chest radiograph and abdominal sonography) were undertaken every 3 months during the first 2 yrs and thereafter every 6 months. CT scans of thorax and cranium were performed at $6,12,18$ and 24 months after final staging.

\section{Immunohistochemistry and histological assessment}

Immunohistochemical assessment of $\mathrm{p} 185^{\text {neu }}$ expression in paraffin-embedded mediastinal lymph node metastases, from biopsies at the primary staging, was performed. Using the alkaline phosphatase/anti-alkaline phosphatase (APAAP) method [15], the primary monoclonal mouse antibody 3B5 (Oncogene Science, Cambridge, MA, USA) was applied. Sections of 3-4 $\mu \mathrm{m}$ were cut, dewaxed and rehydrated through graded alcohols. Sections were incubated for $20 \mathrm{~min}$ at $90^{\circ} \mathrm{C}$ in target-unmasking fluid (TUF) solution (Dianova, Hamburg, Germany) diluted 1:3 in deionized water and then rinsed with Tris-buffered saline (5 mM Tris, $130 \mathrm{mM} \mathrm{NaCl}, \mathrm{pH}$ 7.6). Subsequently, 
sections were reacted with the $3 \mathrm{~B} 5$ primary antibody diluted 1:600 for $24 \mathrm{~h}$ at $4{ }^{\circ} \mathrm{C}$. After rinsing with Tris-buffered saline, sections were stained with monoclonal rabbit antimouse antibody (DAKO, Glostrup, Denmark) for $30 \mathrm{~min}$, followed by incubation for 45 min with APAAP complex according to the manufacturer's recommendations (DAKO). Afterwards, staining-complex solution with neufuchsin was applied for $25 \mathrm{~min}$ and subsequently counterstained with Mayer's hemalaun (Merck, Darmstadt, Germany), washed in distilled water and mounted in glycerol/gelatin (Merck). In each batch, positive (breast cancer tissue) and negative (normal lung tissue) controls were included. Samples were considered positive if $\geq 5 \%$ of the tumour cells on the section showed membrane staining with the antibody. This analysis was performed at the study's reference pathology centre (Institute of Pathology, University Hospital Bergmannsheil, Bochum, Germany) as well as the evaluation of tumour regression in resection samples after surgery. Samples were assigned to the category "tumour regression $>90 \%$ ", when $<10 \%$ tumour cells were present and to the category "tumour regression $<90 \%$ ", if $>10 \%$ tumour cells were present [16].

\section{Statistical analysis}

Statistical analysis was performed using SAS for Windows 6.11 (SAS Institute, Cary, NC, USA). Progressionfree survival curves and survival curves were calculated according to KAPLAN and MEIER [17], using the date of primary diagnosis (primary histology) as a baseline. Subsequently, curves were analysed using the log-rank test [18]. In calculating progression-free survival, progression of the disease or the death of the patient were scored as events. The four-field table Fisher exact test was used to assess the relationship between two variables each with two categories. To estimate the prognostic significance of covariables on progression-free survival, a multivariate analysis was performed by using the stepwise Cox regression model [19]. The significance in all statistical tests was set at $\mathrm{p}<0.05$.

\section{Results}

\section{Response to preoperative therapy and resectability}

The response rate to chemotherapy was $12 / 33$ patients (36\%). One patient (stage IIIA), despite a completely regenerated blood profile, died at the start of the second cycle from primary disease (tumour bleeding due to vessel infiltration) and was scored as local tumour progression. After chemoradiotherapy, the response rate increased to $23 / 33$ patients $(70 \%)$, including four patients with a complete response. One patient had disease progression with brain metastases. Twenty-nine out of 31 patients eligible for surgery, underwent tumour resection and extensive mediastinal lymph node sampling. One patient (stage IIIB) was classified as inoperable during the preoperative restaging, and one patient (stage IIIA) was inoperable for medical reasons. In five patients, resection was incomplete, four had microscopic tumour tissue in the perimeter of the resection samples (R1) and one residual macroscopic tumour in the thorax (R2). The remaining 24 patients $(24 / 33 ; 73 \%), 14$ in stage IIIA and 10 in stage IIIB, were scored R0 (resection margin tumour-free). A discernible tumour regression $>90 \%$ was noted in $20 / 29(69 \%)$ of resection samples (IIIA 12/17; IIIB 8/12 patients). Of the 24 patients who scored R0, 17 displayed a degree of tumour regression $>90 \%$.

\section{Pattern of relapse}

Between diagnosis and the last follow-up, 21 patients experienced disease progression: six had both local progression and distant metastases; seven patients showed exclusively local progress of the disease; and eight had exclusively distant metastases. Metastases were recorded in the brain (three cases), bone (one), lung (three), lymph nodes (two), liver (three) and adrenal glands (two). One patient had multifocal metastases.

\section{Analysis of $185^{\text {neu }}$ expression}

Pretherapeutic $\mathrm{p} 185^{\text {neu }}$ expression was investigated in tumour-bearing paraffin-embedded lymph node biopsies (table 2). Positive staining was detected in eight cases, and seven of these patients developed distant metastases, in contrast to seven out of 25 negative cases. Expression of $185^{\text {neu }}$ in mediastinal lymph node metastases did not depend on the clinical stage (IIIA versus IIIB) or histology (adenocarcinoma versus squamous-cell carcinoma). It was not predictive for a clinical response (complete response (CR)/partial response (PR) versus no change/ progressive disease) to preoperative treatment, resectability (complete ( $\mathrm{R} 0)$ versus incomplete $(\mathrm{R} 1 / 2)$ or no tumour resection) or the degree of tumour regression in resection specimens ( $>90 \%$ versus $<90 \%$ ) (Fisher exact test).

\section{Survival analysis}

With a median follow-up time of 44 months, 10 patients remain alive today, two with and eight without relapse.

Table 2. - Pretherapeutic assessment of $p 185^{\text {neu }}$ overexpression in available mediastinal lymph node biopsies according to stage, histological type and resectability

\begin{tabular}{lcc}
\hline & \multicolumn{2}{c}{ p185 $5^{\text {neu }}$-staining } \\
\cline { 2 - 3 } & Positive & Negative \\
\hline $\begin{array}{l}\text { Biopsies available and } \\
\text { tumour-bearing }\end{array}$ & \multicolumn{2}{c}{$33(28)$} \\
TNM stage & $6(5)$ & $14(11)$ \\
III A & $2(2)$ & $11(10)$ \\
III B & $8(7)$ & $25(21)$ \\
III (total) & $5(4)$ & $19(15)$ \\
Histologic type & $3(3)$ & $6(6)$ \\
Squamous cell carcinoma & $7(6)$ & $17(16)$ \\
Adenocarcinoma & $1(1)$ & $8(5)$ \\
Resectability & \\
R0 &
\end{tabular}

Values are presented as number of patients, with the numbers of patients at risk for recurrence after complete therapy shown in parenthesis. TNM: tumour node metastasis; R0: complete tumour resection with microscopic tumour-free resection margins; Non-R0: comprises patients with unresectable disease or incomplete resection or refusal of surgery. 
Twenty-three patients have died since study entry. In addition to the two therapy-associated fatalities due to pneumonitis and to bronchial stump insufficiency, progression of the disease was the cause of death in 19 patients. Six had distant metastases as well as local disease progression. Six died from distant metastases and seven from local disease progress. Two patients died of causes unrelated to disease progression: one, 23 months postoperatively from a myocardial infarction and the other, 9 months after surgery, from pulmonary embolism.

Median survival of the analysed patients was 22 months with 2- and 3-yr survival rates of $42 \%$ and $28 \%$, respectively (fig. 1). Neither histological subtype (squamous versus adenocarcinoma), age ( $<60$ versus $>60$ yrs), performance score (ECOG 0 versus ECOG 1) nor stage (IIIA versus IIIB) was related to survival (univariate analysis). However, $\mathrm{p} 185^{\text {neu }}$ expression in mediastinal lymph node metastases became apparent as a predictor for progression-free survival ( $185^{\text {neu }}$-positive versus $\mathrm{p} 185^{\text {neu }}$-negative: median, 10 versus 17 months; 2- and 3-yr rates, 13\% and $0 \%$ versus $40 \%$ and $32 \%$, respectively; $\mathrm{p}=0.047$ ). Yet, for survival, this remained without significance $(\mathrm{p}=$ 0.17 ). Shorter progression-free survival of $\mathrm{p} 185^{\text {neu }}$-positive patients resulted mainly from significantly shorter metastases-free survival (fig. 2), whereas $185^{\text {neu }}$ expression had no predictive value for freedom from local progression $(\mathrm{p}=0.21)$. For progression-free survival, a multivariate analysis using performance score (ECOG 0 versus ECOG 1), stage (IIIA versus IIIB) and $185^{\text {neu }}$ expression ( $\mathrm{p} 185^{\text {neu }}$ positive versus $\mathrm{p} 185^{\text {neu }}$ negative) was performed. Whilst performance score and stage were eleminated, $\mathrm{p} 185^{\mathrm{neu}}$ expression maintained an independent predictive value $(\mathrm{p}=0.047)$ for progression-free survival (risk ratio 2.3; 95\% confidence interval 1.1-5.5).

A subanalysis on the impact of $185^{\text {neu }}$ overexpression on progression-free survival was performed on 28 patients who were able to complete the therapy and did not die of any causes other than lung cancer. These patients met the following criteria: 1) patients did not die of therapy-associated fatalities $(n=2)$; 2) patients did not die before therapy was completed $(n=1)$; and 3 ) patients did not die of

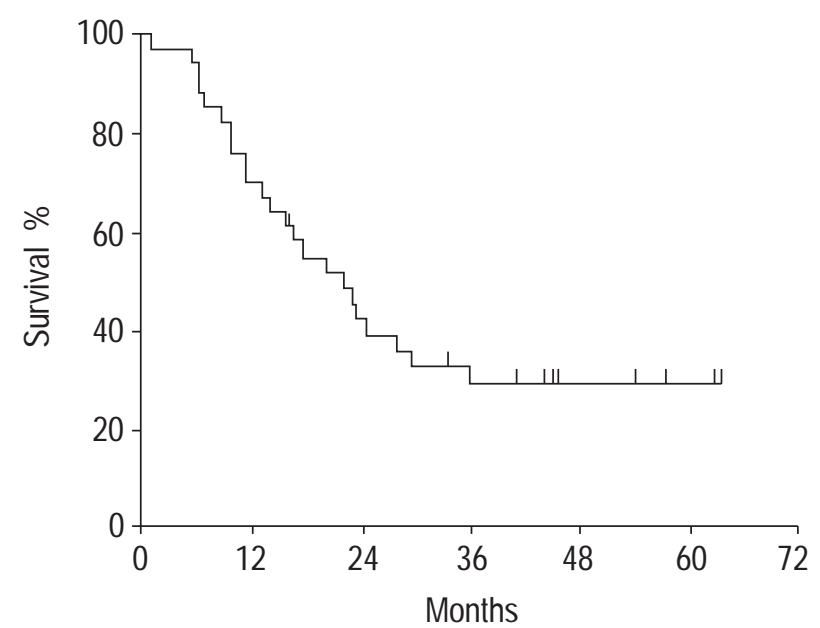

Fig. 1. - Per cent survival from time of diagnosis in patients with paraffin-embedded lymph node metastases available for immunohistochemistry $(\mathrm{n}=33)$. Median, 22 months; 2 - and 3 -year rates, $42 \%$ and $28 \%$, respectively.

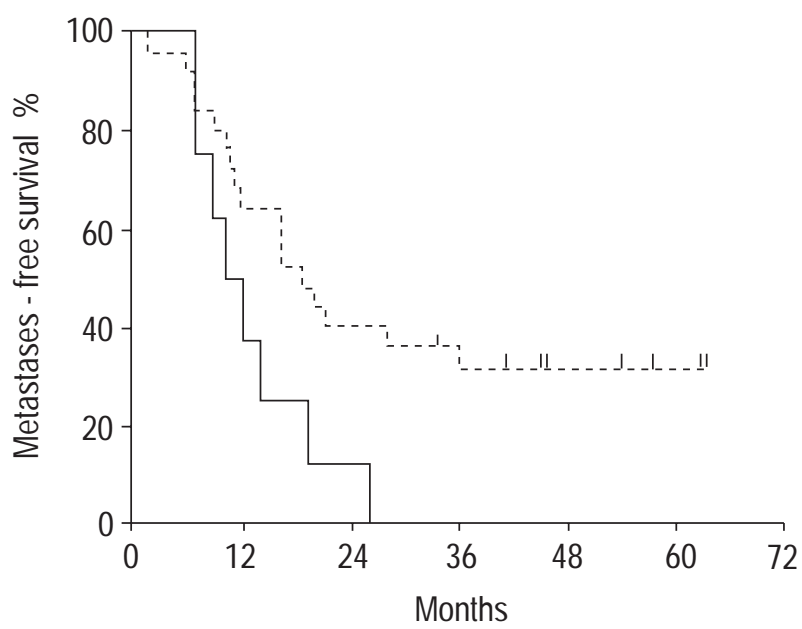

Fig. 2. - Per cent metastases-free survival from time of diagnosis by pretherapeutic $\mathrm{p} 185^{\text {neu }}$ overexpression in patients with paraffin-embedded lymph node metastases available for immunohistochemistry $(n=33)$. Median, 11 versus 19 months; 2- and 3-year rates, $13 \%$ and $0 \%$ versus $40 \%$ and $32 \%$, respectively; $\mathrm{p}=0.04)$. $\longrightarrow$ : 185 positive $(n=8) ;-.-$ : p185 negative $(n=25)$.

any causes other than lung cancer $(n=2)$. Seven of these 28 patients stained positively, and 21 were negative (table 2 ). As seen above for all 33 patients, $\mathrm{p} 185^{\text {neu }}$ expression in mediastinal lymph node metastases was also of predictive value for progression-free survival $(p=0.03)$ and resulted mainly from significantly shorter metastases-free survival $(p=0.02)$. It was not due to any differences in freedom from local progression $(\mathrm{p}=0.15)$.

\section{Discussion}

In the present study, it was demonstrated for the first time that in patients with stage III NSCLC, detection of $\mathrm{p} 185^{\text {neu }}$ overexpression in mediastinal lymph node metastases prior to trimodality therapy was a significant predictor for shorter progression-free survival. This predictive feature resulted mainly from significant differences in metastases-free survival, even with neoadjuvant chemotherapy. In this context in NSCLC, a link between $\mathrm{p} 185^{\text {neu }}$ overexpression and both the enhanced metastatic propensity and the increased intrinsic chemoresistance has to be considered.

Thus, as an indication for enhanced metastatic propensity, a significant correlation between $\mathrm{p} 185^{\text {neu }}$ overexpression in resected squamous cell carcinomas and the occurrence of lymph node metastases has been reported [10]. Furthermore, by comparing metastasis-associated properties between the low $185^{\text {neu }}$ expressing human cell line NCI-H 460 and its c-erbB-2 transfectants, experimental evidence that $\mathrm{p} 185^{\text {neu }}$ enhances metastatic potential in human lung cancer cells has been provided [20]. Thus, the enhanced metastatic propensity of $185^{\text {neu }}$ overexpressing NSCLC is an indication for intensive systemic cytotoxic therapy in these tumours. However, in vitro testing of chemosensitivity in NSCLC cell lines, established from previously untreated patients revealed c-erbB-2 overexpression as a marker for multidrug chemoresistance in encountered cell lines [21]. Moreover, in the cell line NCI-H 460 with low constitutive expression of $185^{\text {neu, }}$ 
significantly enhanced multidrug chemoresistance could be induced by c-erbB-2 transfection [22]. Furthermore, experimental evidence was provided that caffeine enhances the chemosensitivities of high-p $185^{\text {neu }}$ expressing NSCLC cell lines to anticancer agents and that the magnitude of enhancement correlates with the level of $\mathrm{p} 185^{\text {neu }}$ [23]. Caffeine is known to override the cell-cycle arrest triggered by deoxyribonucleic acid (DNA) damaging agents [24], thereby eleminating the presumed opportunity for DNA repair during this arrest [25]. This gives rise to the speculation that, in states of high $\mathrm{p} 185^{\text {neu }}$ expression, for cytotoxic agents interfering substantially with DNA repair, sufficient cytotoxicity could be expected [26]. The link between $185^{\text {neu }}$ overexpression with enhanced metastatic propensity and increased intrinsic chemoresistance in NSCLC cell lines is suggestive of a demand for a highly effective cytotoxic treatment in these cases.

In the present study, pretherapeutic proof of $185^{\text {neu }}$ overexpression in mediastinal lymph node metastases was predictive of a shorter progression-free survival, due to the significantly shorter metastases-free survival of these patients. Given the small sample size and the predominance of squamous cell carcinomas in this patient cohort, the results must be interpreted cautiously. Yet, it can be speculated that in a larger patient population with pretherapeutic $\mathrm{p} 185^{\text {neu }}$ analysis available before trimodality therapy, the same predictive effect will probably become apparent with a significant impact on survival. Indeed, in patients with resectable disease, several reports point to the association of $\mathrm{p} 185^{\text {neu }}$ overexpression with significantly shorter survival $[8,9,11,12]$. A multivariate analysis using p $185^{\text {neu }}$ expression, performance score and stage, revealed $\mathrm{p} 185^{\text {neu }}$ expression in the present study as an independent predictor for progression-free survival. Moreover pretherapeutic p $185^{\text {neu }}$ overexpression did not correlate with clinical stage, histology, clinical response, resectability or degree of tumour regression in resection specimens, even though most of these factors have been shown to be predictive of the survival of NSCLC patients after conventional treatment. This gives rise to the assumption that with larger patient numbers, $\mathrm{p} 185^{\text {neu }}$ overexpression might appear to be a strong independent prognostic factor in the multimodality treatment setting, even more so since, in resectable disease, $\mathrm{p} 185^{\text {neu }}$ overexpression of the tumour has been shown to be an independent prognostic factor for postoperative treatment outcome [8, 11-13]. Additionally it has to be considered that with multimodality treatment, some of these factors mentioned may be no longer of prognostic significance. Thus, in the SWOG phase II trial 8805 [3], with preoperative chemoradiation, the prognostic value of stage, for example, was lost.

The predictive feature of pretherapeutic $\mathrm{p} 185^{\text {neu }}$ overexpression in the neoadjuvant multimodality treatment setting suggests considering this parameter in further trials on neoadjuvant or adjuvant therapy in non-small cell lung cancer. When the prognostic impact of $\mathrm{p} 185^{\text {neu }}$ in such trials with larger patient numbers is confirmed, this may contribute to the identification of stratification variables for future treatment strategies of non-small cell lung cancer. Besides encompassing further tumour-associated prognostic features [5], this approach could possibly be used to withhold ineffective therapy, thus improving the quality of life, or to select patients for a modified and more effective treatment, thus improving their survival. There is experi- mental evidence that selective tyrosine kinase inhibitors preferentially inhibiting $\mathrm{p} 185^{\text {neu }}$ activity may increase the cytotoxicity of chemotherapy and contribute to more effective treatment in non-small cell lung cancer patients with p $185^{\text {neu }}$-positive tumours [27]. Moreover, documented recent reports on $\mathrm{p} 185^{\text {neu }}$ as a biological target for novel treatment strategies with humanized monoclonal antibodies showed the efficacy of this approach in patients with breast cancer expressing high levels of $\mathrm{p} 185^{\text {neu }}[28$, 29]. Although preliminary, these results give rise to the speculation that the adoption of such therapies in patients with $\mathrm{p} 185^{\text {neu }}$ overexpressing non-small cell lung cancer may provide new and effective therapeutic options for this group of patients.

\section{References}

1. Mountain CF. Revisions in the international system for staging lung cancer. Chest 1997; 111: 1710-1717.

2. Johnson DH, Turrisi AT, Pass HI. Combined modality treatment for locally advanced non-small cell lung cancer. In: Pass HI, Mitchell JB, Johnson DH, Turrisi AT, eds. Lung Cancer: Principles and Practice. 1st Ed. New York, Lippincott, 1996; pp. 863-873.

3. Albain KS, Rusch VW, Crowley JJ, et al. Concurrent cisplatin/etoposide plus chest radiotherapy followed by surgery for stages IIIA (N2) and IIIB non-small cell lung cancer: mature results of Southwest Oncology Group phase II study 8805. J Clin Oncol 1995; 13: 1880-1892.

4. Choi NC, Carey RW, Daly W, et al. Potential impact on survival of improved tumor downstaging and resection rate by preoperative twice-daily radiation and concurrent chemotherapy in stage IIIA non-small-cell lung cancer. $J$ Clin Oncol 1997; 15: 712-722.

5. Strauss GM, Kwiatkowski DJ, Harpole DH, Lynch TJ, Skarin AT, Sugarbaker DJ. Molecular and pathologic markers in stage I non-small-cell carcinoma of the lung. $J$ Clin Oncol 1995; 13: 1265-1279.

6. Rusch VW, Klimstra D, Venkatraman E, et al. Aberrant p53 expression predicts clinical resistance to cisplatinbased chemotherapy in locally advanced non-small cell lung cancer. Cancer Res 1995; 55: 5038-5042.

7. Weiner DB, Nordberg J, Robinson R, et al. Expression of the neu gene encoded protein ( $\left.\mathrm{p} 185^{\text {neu }}\right)$ in human nonsmall cell carcinomas of the lung. Cancer Res 1990; 50: 421-425.

8. Kern JA, Schwartz DA, Nordberg JE, et al. p185 expression in human lung adenocarcinomas predicts shortened survival. Cancer Res 1990; 50: 5184-5187.

9. Tateishi M, Ishida T, Mitsudomie T, Kaneko S, Sugimachi $\mathrm{K}$. Prognostic value of c-erbB-2 protein expression in human lung adenocarcinoma and squamous cell carcinoma. Eur J Cancer 1991; 27: 1372-1375.

10. Shi D, He G, Cao S, et al. Overexpression of the c-erbB2/ neu-encoded p185 protein in primary lung cancer. $\mathrm{Mol}$ Carcinogen 1992; 5: 213-218.

11. Kern JA, Slebos RJ, Top B, et al. c-erbB2 expression and codon $12 \mathrm{k}$-ras mutations both predict shortened survival for patients with pulmonary adenocarcinoma. J Clin Invest 1994; 93: 516-520.

12. Harpole DH, Herdon JE, Wolfe WG, Iglehart JD, Marks JR. A prognostic model of recurrence and death in stage I non-small cell lung cancer utilizing presentation, histopathology and oncoprotein expression. Cancer Res 1995; 55: $51-56$. 
13. Diez M, Pollan M, Torres A, et al. Prediction of recurrence by quantification of $\mathrm{p} 185$ neu protein in non-smallcell lung cancer tissue. Br J Cancer 1997; 75: 684-689.

14. Green S, Weiss GR. Southwest Oncology Group standard response criteria, endpoint definitions and toxicity criteria. West New Drugs 1992; 10: 239-253.

15. Cordell JL, Falini B, Erber WN, et al. Immunoenzymatic labeling of monoclonal antibodies using immune complexes of alkaline phosphatase and monoclonal anti-alkaline phosphatase (APAAP-complexes). $J$ Histochem Cytochem 1984; 32: 219-229.

16. Junker K, Thomas M, Schulmann K, Klinke F, Bosse U, Müller KM. Tumor regression in non-small cell lung cancer following neoadjuvant therapy - histological assessment. J Cancer Res Clin Oncol 1997; 23: 469-477.

17. Kaplan EL, Meier P. Non-parametric estimation from incomplete observations. J Am Stat Assoc 1958; 53:457-481.

18. Peto R, Pike MC, Armitage P, et al. Design and analysis of randomized clinical trials requiring prolonged observation of each patient. II analysis and examples. $\mathrm{Br} J$ Cancer 1977; 35: 1-39.

19. Cox DR. Regression models and life tables (with discussion). J R Stat Soc B 1972; 34: 187-220.

20. Yu D, Wang SS, Dulski KM, Tsai CM, Nicolson GL, Hung MC. c-erbB-2/neu over-expression enhances metastatic potential of human lung cancer cells by induction of metastasis-associated properties. Cancer Res 1994; 54: 3260-3326.

21. Tsai CM, Chang KT, Perng RP, et al. Correlation of intrinsic chemoresistance of non-small-cell lung cancer cell lines with HER-2-neu gene expression but not with ras gene mutations. J Natl Cancer Inst 1993; 85: 897901.

22. Tsai CM, Yu D, Chang KT, et al. Enhanced chemoresistance by elevation of $\mathrm{p} 185^{\text {neu }}$ levels in HER-2/neu- transfected human lung cancer cells. $J$ Natl Cancer Inst 1995; 87: 682-684.

23. Tsai CM, Perng RP, Chen MH, et al. Greater enhancement of chemosensitivity by caffeine in high-p $185^{\text {neu }}$ expressing human non-small-cell lung cancer cell lines. $J$ Natl Cancer Inst 1994; 86: 1018-1020.

24. Schlegel R, Pardee A. Caffeine induced uncoupling of mitosis from the completion of DNA replication in mammalian cells. Science 1987; 232: 1264-1266.

25. Lock RB, Ross WE, Mattern J. Inhibition of $\mathrm{p} 34^{\text {cdc2 }}$ kinase activity by etoposide or irradiation as a mechanism of $\mathrm{G}_{2}$ arrest in Chinese hamster ovary cells. Cancer Res 1990; 50: 3761-3766.

26. Tsai CM, Chang KT, Chen JY, Chen YM, Chen MH, Perng RP. Cytotoxic effects of gemcitabine-containing regimens against human non-small cell lung cancer cell lines which express different levels of $185^{\text {neu }}$. Cancer Res 1996; 56: 794-801.

27. Zhang L, Hung MC. Sensitisation of her-2/neu-overexpressing non-small cell lung cancer cells to chemotherapeutic drugs by tyrosine kinase inhibitor emodin. Oncogene 1996; 12: 571-576.

28. Cobleigh MA, Vogel CL, Tripathy D, et al. Efficacy and safety of herceptin (humanized anti-HER2 antibody) as a single agent in 222 women with HER2 overexpression who relapsed following chemotherapy for metastatic breast cancer. Proc Am Soc Clin Oncol 1998; 17: 97 (abstract No. 376).

29. Slamon D, Leyland-Jones B, Shak S, et al. Addition of herceptin (humanized anti-HER2 antibody) to first line chemotherapy for HER 2 overexpressing metastatic breast cancer (HER2+/MBC) markedly increases anticancer activity: a randomized multinational controlled phase III trial. Proc Am Soc Clin Oncol 1998; 17: 98 (abstract No. 377). 